\title{
La identidad nacional en tiempos de globalización
}

Recibido 30 de julio de 2008 • Aceptado 29 de abril de 2009

“...la identidad no es una pieza de museo quietecita en una vitri na, sino la siempre asombrosa síntesis de las contradicciones nuestras de cada día".

Leonel Arias Sandoval

Académico de la licenciatura en Pedagogía en la División de Educología, Centro de Investigación y Docencia en Educación, Universidad Nacional

Heredia, Costa Rica

Eduardo Galeano, 1989

\begin{abstract}
Resumen. La identidad nacional es un proceso histórico y geográfico, dinámico y en constante transformación, en otras palabras: está sujeta al cambio. Es construida por los individuos y diferentes grupos sociales que nacen o viven en un territorio mediante el discurso ideológico homogenizador y reproductor del imaginario nacional y, por otra parte, la influencia cultural expresada por las interrelaciones con otros países, la cual se ha acelerado por medio del fenómeno de la globalización. Este último aspecto tiene un impacto profundo no sólo en el campo económico, sino, también, en el intercambio de información, ideas, valores y comportamientos que asimilan las personas por medio de conductas, símbolos y significados que tienen que ver con la imitación de modelos culturales foráneos. Pero existe un punto en común en el contexto de este ensayo, y es entender que estamos ante un proceso evolutivo, dinámico y complejo, que forma parte de los procesos de socialización que vinculan las prácticas cotidianas de los individuos, mediante la diversidad y el intercambio cultural.
\end{abstract}

Palabras clave. Identidad, globalización, cultura, educación, multiculturalidad, diversidad, valores, socialización.

\footnotetext{
${ }^{1}$ Magíster en Psicopedagogía por la Universidad Estatal a Distancia. Licenciado en Ciencias de la Educación con Énfasis en Didáctica por la Universidad Nacional de Costa Rica. Bachiller en la Enseñanza de los Estudios Sociales por la Universidad Nacional de Costa Rica. Actualmente, forma parte del equipo de académicos de la Licenciatura en Pedagogía en la División de Educología de la Universidad Nacional de Costa Rica y Coordinador Académico del Liceo el Carmen de Alajuela.

Correo electrónico: ariasleonel@yahoo.com
} 


\begin{abstract}
The national identity is a historical and geographical process; it is also dynamic and it is constantly transforming, in other words it is: a subject of change. It is constructed by individuals and different social groups who are born or live in a territory with a homogenizing ideological discourse and a reproducer of an imaginary national and, moreover, the cultural influence is expressed by the interrelationships among other countries, which have been accelerated by the phenomenon of globalization.

This last aspect has a profound impact not only in the economic field, but also when sharing information, ideas, values and behaviors that are assimilated by people through behaviors, symbols and meanings that are related to the imitation of foreign cultural models. But there is one point in common in the context of this essay and it is to understand that we are in an evolutionary, dynamic and complex process that is part of the socialization processes that link the daily practices of individuals, through the diversity and cultural exchange.
\end{abstract}

Key words. Identity, globalization, culture, education, multiculturalism, diversity, values, socialization.

\title{
Consideraciones preliminares
}

En la actualidad, el mundo es diferente, está en constante cambio, y los eventos que ocurren cotidianamente, en nuestro país, están relacionados con situaciones que se presentan en otros lugares del planeta. La globalización tiene responsabilidad en este fenómeno, ya que no sólo abarca aspectos económicos, sino, también, políticos, sociales y hasta culturales; los cuales forman parte de lo que ocurre en la realidad; pero cada vez con mayor intensidad y alcance.

Los cambios observados en el transcurso de los últimos años, con los avances tecnológicos, el incremento de las relaciones comerciales, los movimientos migratorios y las transformaciones político-económicas, han provocado no sólo una mayor integración global, sino una relativa interdependencia que alcanza, prácticamente, todos los aspectos de la vida social: la economía, la política, la ecología, la comunicación, la cultura e, incluso, el fenómeno identitario.

La globalización acentúa los procesos de cambio social, fenómeno mediante el cual los medios de comunicación masiva, tales como la televisión por cable, satélite, Internet y otros, juegan un importante papel en el intercambio de información y flujo de ideas, conocimientos, valores y, por consiguiente, el entorno cultural se transforma aceleradamente. Desde este punto de vista, a éstos “....se les responsabiliza de promover estereotipos errados de las condiciones de joven, inducir valores y prácticas foráneas que dificultan el desarrollo de las identidades, promover prácticas consumistas, egoístas, masificadotas y superficiales...” (Garro, 2002, p. 27).

Por tanto, la influencia de los medios de comunicación debe verse como un eje problematizador en la construcción de las identidades nacionales y no solamente de aquellas posiciones que tienden a responsabilizarlos "... de homogenizar las mentalidades de sus audiencias al ofrecer a todos los individuos los mismos contenidos" (Garro, 2002, p. 27). Al respecto, Buckingham, citado por Garro, señala que "...existen pocos intentos de investigar la forma en que, por ejemplo, los jóvenes, usan los medios y los significados y valores que derivan de ello. Más bien se interpreta que en dicha relación estos son víctimas pasivas e independientes de los efectos negativos de los medios de comunicación" (p. 27).

Desde esta perspectiva, observamos que nuestro país se integra cada vez más dentro del contexto mundial de la interdependencia, con la intensificación de relaciones más abiertas y activas con la comunidad internacional. Este proceso le da mayor vigencia a la necesidad de analizar tal fenómeno desde una óptica valorativa, con base en los nuevos vínculos con otros países y culturas. Asimismo, se da una relación, de manera más estrecha, con las exigencias de una sociedad que cambia y cambiará intensamente en los próximos años.

Para algunos sectores sociales, el fenómeno de la globalización cultural incide en la denominada crisis de identidad, ya que han sido adoptadas actitudes y conductas ajenas, por la 
influencia de la industria cultural ${ }^{2}$ y de los medios de comunicación. Abarca (2001) afirma que “... durante las últimas décadas, gran parte de la sociedad costarricense, se encuentra sometida a un acelerado proceso de deformación de sus hábitos, costumbres y actitudes" (p. 9). Desde este punto de vista, la globalización se presenta como un proceso unificador al interior de las naciones. No obstante, Leandro (2002) plantea que la realidad ha reflejado una serie de contradicciones al interior de los Estados Nacionales, lo cual ha propiciado prácticas discriminatorias hacia ciertos grupos marginados de la sociedad, y considera que

...a pesar de que el discurso de la globalización se presenta como unificador (de economías, mercados, fronteras, entre otros), en la realidad suceden situaciones bastante distintas, los procesos de xenofobia y de discriminación social en general se agudizan y cada vez se convierte más en un mundo polarizado donde sólo existen los excluidos y los incluidos, aunque ambos lugares sean, y quizá esto resulte paradójico, globalizados. (p. 52)

El discurso de la homogenización cultural, basado en principios tales como el individualismo y el consumismo, encuentra en la población joven uno de los sectores más receptivos a las nuevas ideas. Pero los intercambios que se dan por medio del proceso de interacción social ${ }^{3}$, no necesariamente deben verse a la luz de una relación pasiva; esto por cuanto se considera que la juventud participa y aporta en esa relación, gracias al proceso de socialización.

Entonces, si bien es cierto que, dentro de la contextualización histórica del surgimiento de la globalización, lo económico es importante, ésta no es la única variable que debe tomarse en cuenta, pues existen otros elementos importantes como lo social, lo político y lo cultural que inciden en la construcción de los procesos identitarios. Ante esta situación histórica, que nos muestra una integración, es necesario analizar la posición del joven en toda esta maraña de cambios, ya que si se quiere determinar los retos del educador en el contexto de la globalización, se debe tener claro el mundo y los problemas que enfrenta la juventud de inicios del siglo XXI.

\section{Cultura, educación y juventud}

A través de la historia, la educación ha jugado un rol conservador en la conformación de una identidad nacional, en el marco del discurso ideológico esencialista, hegemónico y reproductor de ciertas normas, y ciertos ritos y valores de carácter nacionalista. Pero, según Denis (1995), el aspecto formativo no sólo debe enfocarse en el resguardo de ciertos valores sociales, sino que "...el mismo proceso educativo debería ser lo suficientemente flexible y dinámico como para aceptar la realidad cambiante del sistema valorativo de la sociedad o de cada grupo humano" (p. 3). Por consiguiente, primero hay que comprender el entorno sociocultural en que vivimos y, luego, propiciar alternativas hacia la formación de personas humanistas, respetuosas del ambiente, y tolerantes de las prácticas culturales de otros grupos humanos y del ambiente en que vive y convive cotidianamente.

Es claro que los procesos identitarios responden a un contexto específico, en el que los individuos y los grupos comparten distintos espacios sociales, por medio de su interacción comunicativa, sus

\footnotetext{
${ }^{2}$ El término es empleado por primera vez por la Escuela de Frankfurt, y se refiere a la producción de estrategias para crear objetos o símbolos culturales, con el fin de ser consumidos.

${ }^{3}$ Proceso dialéctico, por el que se da un intercambio cultural y en el cual los sujetos reciben y, al mismo tiempo, aportan a la estructuración del medio en que viven.
} 
comportamientos y los símbolos culturales. Esos espacios son sitios de dominación y resistencia, de conformismo y oposición, de subordinación o crítica; por eso se afirma que la escuela “...es un sitio cultural y político que representa un espacio de contestación y lucha entre grupos diferentes en el que se construyen y reconstruyen los contenidos culturales y las relaciones sociales" (Denis, 1995, p. 5).

Por ello, se impone la necesidad de analizar en el aula los elementos valorativos que subyacen en los comportamientos y significados de la juventud y que tienen estrecha conexión con las prácticas culturales que incorporan en su cotidianidad. Entonces, es necesario no sólo observar o caracterizar dichos cambios, sino, también, reflexionar sobre la responsabilidad que tiene el maestro para practicar una educación inclusiva, vivencial y respetuosa de la diversidad. En este sentido, uno de los conceptos actuales de aula es el referido a aquel espacio donde se construye una pluralidad de saberes con sentido y significado cultural.

Posiblemente, el mundo actual no es fácil de entender para la juventud. Esto por cuanto los intereses económicos mundiales de una sociedad globalizada, quieren a un joven absorto del mundo real, que tenga como prioridad el consumo y no la creación. Ello plantea una visión subjetiva, estereotipada por las telenovelas, los juegos de vídeo, la Internet; los cuales los convierten en un objetivo de mercado y les ofrece sexo, violencia, drogas, modas, entre otras manifestaciones sociales, para estar a tono con la época, haciéndoles creer que lo pasado es obsoleto.

Pero, para Achugar, citado por Rivera (1997), la construcción de las identidades no es un tema de simple análisis, ya que por ejemplo “...el llevar una camiseta con el logo de Guns N' Roses, escuchar rock extranjero o, bien, comer hamburguesas en Burger King, no quiere decir que no estén identificados con su país" (p. 8). Entonces, no se puede medir la identidad nacional sólo con este tipo de parámetro, lo que falta es comprender la dinámica del cambio social.

El aula constituye uno de los lugares más apropiados para reconocer la forma en que los y las estudiantes, como seres pertenecientes a una comunidad, se identifican con ella o no; pues ahí, tanto educandos como educadores, comparten no solamente conocimientos, sino, además, valores y actitudes que forman parte de su identidad. Asimismo, los jóvenes reciben diferentes aportes culturales que los identifican y, a la vez, los diferencian de los demás grupos sociales.

\section{Identidad nacional: contextualización histórico-geográfica}

La identidad es producto de un proceso que se ha construido y reconstruido a lo largo del tiempo y que tiene estrecha relación con las particularidades culturales propias de cada región geográfica y de las características que identifican a los pueblos en un momento histórico determinado. En esta construcción, la geografía tiene correspondencia con la historia, para interpretar y entender el pasado de un pueblo en relación con los elementos espaciales, sociales, económicos, políticos y culturales que se han modificado a través del tiempo. Meléndez (2004) afirma que “...la geografía, conjuntamente con la historia, ha permitido el conocimiento del territorio y del pasado, elementos indispensables en los procesos de construcción nacional. Todo eso se puede observar tanto en los libros geográficos como en los utilizados en la enseñanza de las disciplinas, como también en el material cartográfico" (p. 81).

Sobre este particular, este autor señala que el surgimiento de una identidad nacional en Costa Rica ha sido parte de un proceso gradual que se caracterizó por el surgimiento del EstadoNación y la delimitación de las fronteras políticas, lo que fortaleció el carácter de pertenencia a un determinado territorio; además de la construcción de un discurso político-ideológico 
por parte de las elites dominantes por medio de un modelo homogenizador, fortalecido por el estado a través de la educación y que, a la fecha, ha permitido mantener un imaginario nacional "...que a su vez evoluciona y cambia diariamente, para mantener su relevancia y significado" (Meléndez, 2004, p. 27).

La posición de una construcción histórico-geográfica del proceso identitario es cohesionada por la cultura de cada pueblo, su manera de vivir y de socializar. Entonces, cabe indicar que la identidad nacional permite "... a los miembros de un grupo social que compartan una historia y un territorio común, así como otros elementos socioculturales, tales como un lenguaje, una religión, costumbres e instituciones sociales...". (Leandro, 2002, p. 54).

\section{Identidad Nacional ¿Conservación o Cambio?}

Desde un punto de vista conservador, podría decirse esencialista, la identidad nacional alude a un sentido de pertenencia que trasciende, más allá de lo afectivo. Altamirano (1997) hace referencia a la obligación que tenemos todas las personas de salvar lo nuestro, y afirma que "...preservar gustos, costumbres y valores nacionales y afirmar y elevar el carácter del ser costarricense, es la forma más sensata y efectiva de contribuir a preservar la identidad cultural de cada pueblo, de cada país" (p. 89). Añade que se debe valorar lo criollo, lo nativo, lo folclórico, lo autóctono, lo nacional; para afirmar la idiosincrasia de nuestra cultura, garantizar la continuidad y coherencia del universo genuinamente costarricense. Este discurso de lo nacional, de costumbres, tradiciones y valores propios es lo que ha definido el concepto de identidad nacional.

De igual manera, Carazo (1997) afirma que “...para sobrevivir como nación hay que utilizar el lenguaje de la patria, más pragmático y más simple, más vivencial y más integrador” (p. 127). En este contexto se utiliza el término patria para sustentar un discurso reproductor y nacionalista, en el cual la institucionalidad, la familia, la religión y la educación se ven amenazadas ante el cambio y, por tanto, hay que preservarlas. De igual manera, Zelaya, (1991) sostiene que la identidad nacional es “....apreciar la vida nacional, el espíritu y la fisonomía de la nación. Respetar a la persona y su tierra, valorar nuestra idiosincrasia. Sentirse orgulloso de nuestras tradiciones" (p. 7).

Se puede inferir que la posición tradicional de la identidad nacional tiene un tono esencialista, enmarcado por las costumbres, las tradiciones, las instituciones sociales, la familia, la religión y, por otro lado, elementos ideológicos como la política y la educación. Los anteriores planteamientos se unen a la idea del discurso de lo nacional, de la esencia del ser.

Es necesaria la reflexión sobre el tema de la identidad nacional, en cuanto a las posiciones del discurso tradicional o conservador en el que "...es frecuente hablar de identidades primordiales o auténticas, (...) como partícipes de ciertas características esenciales, inmutables y en consecuencia ahistóricas" (Téllez y López, 2002, p. 21). Estos autores afirman que

...los riesgos de tales enfoques son cada vez más evidentes y conducen, en primer lugar, a explicaciones que no logran evidenciar los elementos que integran a los procesos identitarios (...). Cuando se pierde de vista este tipo de situaciones, se termina por hacer abstracción de los elementos del pasado. Aparecen conceptos como "lo único", "lo verdadero" o "lo esencial" de las identidades (...). Cualquier cultura que se define a partir de una supuesta "autenticidad", se sitúa en oposición radical a cualquier otra (...). Los procesos de exclusión, marginación, segregación e incluso de "purificación” étnica o nacional descansan sobre tales concepciones... (p. 22) 
Por lo anterior, debemos preguntarnos: ¿es la identidad nacional una realidad estática, inmutable? Esta pregunta tiene varios significados, ya que algunas posiciones se refieren a un proceso social, dialéctico, en constante construcción, que se contrapone a la visión ideológica, nacionalista y conservadora. Por ejemplo, para Induni (2002, p. 69) “...la identidad no es un estado, sino más bien un proceso. Por consiguiente, no puede ser aprendida sino dentro de la lógica del cambio social. Por eso, la identidad es un fenómeno sociocultural y como tal histórico y político". Esta posición, que alude al cambio social, permite establecer la existencia de una serie de significados que las personas poseen, por lo que las diferencias sociales permiten deducir que lo que es significativo para un grupo, puede que no lo sea para otro. Por tanto, la identidad "...tiene que ver con la inserción de las personas en un determinado mundo de significaciones y en una determinada red de relaciones sociales..." (Leandro, 2002, p. 55). Entonces, es en el proceso de socialización donde nace y se vivencia nuestra identidad, pues es ahí donde se da la construcción de los significados, “...las condiciones materiales y simbólicas a partir de las cuales se fundan las identidades" (Induni, p. 55).

En el proceso de construcción de una identidad, Leandro (2002), distingue dos aspectos fundamentales: el altercentrismo y el etnocentrismo. El primero se relaciona con la dependencia, la cual considera que, lo mejor viene de afuera, por lo que las personas adoptan modelos, valores, actitudes y otras manifestaciones culturales externas, ya que son consideradas superiores por el grupo social. El segundo se refiere a "...aquellas formas de entender, sentir y actuar que asumen como único criterio válido los principios del propio grupo, mientras rechazan y condenan cualquier otra forma de entender y sentir y actuar ante la realidad" (Leandro, p. 56).

Entendida la socialización como proceso, no es de extrañar que las personas asuman valores hegemónicos y contradictorios dentro de la sociedad, exigiéndole nuevos comportamientos y actitudes. Es así, que la educación cambia de acuerdo con las transformaciones sociales que la misma sociedad enfrenta. Entonces,

...el proceso de socialización que las nuevas generaciones soportan tanto en su entorno social como en la escuela cambian y se especializa a la medida y ritmo de las sutiles y aceleradas transformaciones sociales. Por ejemplo, la ideología postmoderna que corresponde a la estructura económica del liberalismo radical del mercado está transformando de forma acelerada valores y actitudes aparentemente bien asentados en las sociedades llamadas modernas y occidentales. (Pérez, 1997, p. 46)

Otros autores expresan, con respecto a la identidad, que “...la discusión sobre este tema reconoce cada vez más -a pesar de opiniones contrarias- que éstas más que ser estáticas y tender a la homogenización, tienen una tendencia hacia su restructuración y recomposición permanentes" (Téllez y López, 2002, p. 19). Lo anterior, es producto del intercambio cultural y social, así como de la apropiación de elementos externos que se encuentran en los diversos grupos que conforman la sociedad o bien a través de la industria cultural. A su vez, “...la idea de que las tradiciones, culturas, identidades y diferencias son representaciones simbólicas socialmente construidas- y no legados pasivamente heredados-ha venido recientemente el foco de convergencia de relevantes estudios" (Mato, 1994, p. 16).

Es de entender que, estamos inmersos en un mundo cambiante, en el cual los diferentes puntos de vista, forman parte de los procesos identitarios. Para García (1999), “...la globalización debe verse como un fraccionamiento articulado del mundo, que reordena las diferencias y las desigualdades sin suprimirlas (p. 49). Para Téllez y López (2002), “...este proceso se da 
normalmente a través de mecanismos que implican préstamos o reapropiación de elementos de organización social y cultural que tienen con frecuencia un origen externo a los grupos humanos que se estudian (p. 19).

Lejos de exponer aspectos subjetivos, la posición de la heterogeneidad muestra que los procesos globalizantes brindan a las identidades nacionales la oportunidad de trabajar con su diversidad. Al respecto, García (1999) expresa que “...las mismas empresas transnacionales, como la Coca Cola y Sony están convencidas de que la globalización no significa construir fábricas por todo el mundo, sino en convertirse en parte viva de cada cultura..." (p. 51). De esta manera, al compartir con otras identidades, nos relacionamos con otras culturas y al hacerlo se debe tener presente que esos rasgos que identifican e integran, lejos de discriminar o ser discriminados; debe propiciar ambientes para el cruce e intercambio cultural, lo cual ha sido la constante a través de la historia de la humanidad.

Ante la homogenización y heterogenización, establecida dentro del discurso de la globalización, es importante destacar la posición de Giroux (1992), quien considera que la identidad “...ya no puede ser escrita bajo los lentes de la uniformidad cultural o impuesta mediante el discurso de la asimilación. Ha emergido una nueva cultura postmoderna basada en la especificidad, la diferencia, la pluralidad y los discursos múltiples" (p. 109). Desde esta perspectiva, García (1999), supone que en el proceso de globalización coexisten, tanto procesos de homogenización, como de heterogenización, por cuanto

...si no contamos con una teoría unitaria de la globalización no es sólo por deficiencias en el estado actual del conocimiento, sino también porque lo fragmentario es un rasgo estructural de los procesos de globalización. Para decirlo más claro, lo que suele llamarse globalización se presenta como un conjunto de procesos de homogenización y, a la vez, de fraccionamiento articulado del mundo, que reordenan las diferencias y las desigualdades sin suprimirlas. (p. 49)

De esta manera, lo nacional, desde el punto de vista de la heterogeneidad, no debe reñir, necesariamente, con lo extraño, sino que al vincularnos con otras identidades, nos relacionamos con nosotros mismos. Se debe tener presente que los rasgos culturales que identifican e integran, lejos de discriminar o ser discriminado, propician ambientes para el cruce e intercambio cultural. Por consiguiente, estamos ante un proceso histórico innegable, dinámico y complejo; por ello hay que reflexionar acerca de cuáles identidades fomenta la sociedad y, también, acerca de cuáles, inevitablemente, perjudica.

\section{Multiculturalidad y globalización}

Los procesos identitarios llevan explícita una discusión en torno a la dinámica de cambio, el papel de los grupos sociales emergentes y la diversidad sociocultural, y que, paulatinamente, el discurso esencialista e inmutable de las identidades ha sido cuestionado. Desde esta perspectiva, es claro que los distintos grupos humanos van construyendo y reconstruyendo identidades a la par de la influencia que ejerce la industria cultural, ya sea por los flujos migratorios, los medios de masas o la actividad comercial, en una relación interactiva que da paso a varios fenómenos sociales, entre ellos:

1. La aparición de espacios sociales, en los que se promueven prácticas culturales alternativas y emergentes. 
2. La promoción de la diversidad y el reclamo de las minorías por sus derechos.

3. La dinámica de los micromundos dentro de la concepción política de Estado.

4. La presión política de los organismos internacionales por los derechos humanos.

5. El surgimiento de la cultura popular ${ }^{4}$.

6. La pluralidad de expresiones culturales enmarcadas en símbolos, modas, valores, conocimientos, ideas y prácticas sociales de diversa naturaleza.

Todos estos cambios son el resultado de un nuevo orden en la conformación de las identidades, en la era de la globalización cultural. Por consiguiente, se afirma que

...la globalización trae consigo una mayor conciencia de las diferencias entre identidades culturales, sea porque se difunden en los medios de comunicación de masas, se incorporan al nuevo imaginario político difundido por ONGs transnacionales, o se intensifican las olas migratorias; o sea, porque hay culturas que reaccionan violentamente ante la ola expansiva de la "cultura-mundo" y generan nuevos tipos de conflictos regionales que inundan las pantallas en todo el planeta. De este modo, aumenta la visibilidad política del campo de la afirmación cultural y de los derechos de la diferencia. (Hopenhayn, 2002, p. 1)

Dentro de cada cultura, coexisten distintos modos de vivir; entonces, se habla de multiculturalidad cuando en el seno de una unidad socio-política se vive de acuerdo con diferentes opciones culturales. En este caso, coexisten distintos modos de vida y expresiones culturales, de acuerdo con las distintas adaptaciones a las condiciones geográficas, económicas, políticas y educativas (pueblos, etnias, grupos regionales, grupos ocupacionales, rurales, urbanos, lingüísticos, religiosos, la cultura popular, entre otros). Se habla de multiculturalidad cuando en el seno de una unidad socio-política se puede vivir de acuerdo con diferentes opciones culturales y a la diversidad que ésta ofrece.

\section{Consideraciones finales}

Por las distintas posiciones y discursos, es necesario asumir que existen diversas interpretaciones en torno a si existe o no una única identidad, ante el impacto del fenómeno de la globalización. Incluso, hoy día se habla de identidades transnacionales, definidas como "... aquellas que vinculan a grupos étnicos y/o de otro tipo, a través de las fronteras de los Estado- Nación” (Mato, 1994, p. 254). Por tanto, existen diversas identidades que se construyen mediante los procesos de socialización, por lo que ésta no es estática, ya que los sistemas sociales han introducido elementos culturales muy diversos, con un mundo de significados y representaciones simbólicas propias o impregnadas por la industria cultural, a través de los medios de comunicación. Por tanto, el discurso y el debate todavía están en proceso de construcción, por lo que hay que adoptar una posición integral del fenómeno social, que se vive actualmente.

Es de considerar, entonces, que el proceso de globalización no sólo debe verse como el auge de las nuevas tecnologías de la comunicación o de la ampliación de mercados. Es un proceso más complejo, que trasciende lo económico y tecnológico. Las tendencias actuales giran en torno a la

\footnotetext{
${ }^{4}$ Cultura de los grupos sociales no hegemónicos. En el caso de Costa Rica, comprende la cultura de los indígenas, los afrocaribeños, los campesinos y distintos grupos sociales urbanos. Ciertas posiciones teóricas (Antonio Gramsci) le atribuyen contenido revolucionario, de oposición y resistencia, frente a los valores culturales hegemónicos.
} 
interrelación de diversos factores políticos, sociales y culturales, que se observan en los símbolos y significados de los diversos grupos sociales. Por consiguiente, la identidad nacional es un proceso histórico, dinámico y en constante transformación; en otras palabras, está sujeta al cambio, pues forma parte de los procesos de socialización que vinculan las prácticas cotidianas de los individuos y los grupos sociales gracias a la diversidad e intercambio cultural, de los grupos sociales que nacen y/o viven en un territorio. Además,

...ahora, tenemos un reto muy bonito, para evitar que nos conviertan en los esclavos anónimos de la economía de mercado(...) propongo que contribuyamos a la construcción de una cultura nacional ampliando las fronteras ticas más allá de las montañas de la meseta, abriendo espacios a los grupos periféricos y marginales, al sector afrocaribeño, al indígena, al guanacasteco... (Lobo, 1998, p. 3).

De las anteriores reflexiones, queda claro que la identidad nacional es un tema que se encuentra en discusión, a partir de diferentes tendencias ideológicas, por lo que una característica del fenómeno de las identidades es la diversidad en cuando al discurso que se brinda. Por una parte, se habla de "crisis de identidad" como algo negativo, podría decirse ruptura con lo establecido, de aquella posición ideológica conservadora y/o reproductora del discurso de "nosotros" frente a los "otros", del sentimiento nacionalista del ser costarricense, de lo nuestro, de narraciones pasadas, entre otros aspectos de carácter evidentemente esencialista. Contrario a esta postura, está la visión de cambio social y transformación de lo propio, a causa de la influencia de los medios de comunicación masiva, principalmente de la televisión y la Internet.

Por ello, existe un punto en común en el contexto en que este debate se realiza y es que tal proceso tiene relación directa con la globalización. Este aspecto es fundamental para entender la dinámica de cambio social que vivimos en estos tiempos, en la que lo foráneo viene a convertirse en un factor determinante para diferenciar, compartir o afirmar una forma de identidad. Además, es una construcción social que se reconoce al interior de cada sociedad, en aspectos tales como el género, los grupos étnicos, el lugar en donde se nace o se vive, el aspecto generacional, la edad, las diferencias socioeconómicas, las tendencias políticas e, incluso, las preferencias sexuales; por lo que la diversidad se convierte en una característica que cohesiona el fenómeno de las identidades en tiempos de globalización.

\section{Referencias bibliográficas}

Abarca D., M. (2001). Fruto de la perseverancia. Sistema Nacional de Comisiones de Valores de Costa Rica. San Pedro, Costa Rica: Editorial de la UCR.

Altamirano, C. (1997). Los símbolos nacionales de Costa Rica. San José, Costa Rica: Imprenta Los Sauces.

Carazo, M. (1997). Nuestra Identidad Nacional. Revista Parlamentaria No 2, Volumen 5, pp. 127-132.

Denis, L. (1995). Investigación cualitativa sobre valores en el aula. Recuperado el 22 de marzo de 2008, de www.tripot.com/denissantana/tesis/index.html 
Galeano, E. (1989). El libro de los abrazos. Buenos Aires, Argentina: Siglo XXI.

García C., N. (1999). Globalización imaginaria. Buenos Aires, Argentina: Paidós.

Garro, L. (2002). Escudriñando la relación entre tiempo libre y medios de comunicación. Jornada de Investigación sobre juventud. Consejo Nacional de Política Pública de la Persona Joven. San José, Costa Rica: Ministerio de Cultura, Juventud y Deportes.

Giroux, H. (1992). Nuevas perspectivas críticas en educación. Barcelona, España: Paidós.

Hopenhayn, M. (Febrero, 2002). El reto de las identidades y la multiculturalidad. Pensar Iberoamérica, número 0. Recuperado el 25 de mayo de 2009, de http://www.oei.es/pensariberoamerica/ric00a01.htm

Induni, G. (2002). Juventudes y construcción de identidades sociales: el papel de lo nacional. San José, Costa Rica: Ministerio de Cultura, Juventud y Deportes.

Leandro, V. (2002). Juventudes y construcción de identidades sociales: el papel de lo nacional. San José, Costa Rica: Ministerio de Cultura, Juventud y Deportes.

Lobo, T. (1998). Por una identidad nacional, libre y autogestionaria. Suplemento cultural N ${ }^{\circ} 51$, Programa ICAT, UNA.

Mato, D. (1994). Teoría y política de la construcción de identidades y diferencias en América Latina y el Caribe. UNESCO, Caracas, Venezuela: Editorial Nueva Sociedad.

Meléndez, S. (2004). Aportes geográficos al imaginario costarricense en el siglo XIX. Revista Reflexiones 83 (1), pp. 57-85.

Pérez Gómez, A. I. (1997). Socialización y educación en la época posmoderna. En Ensayos de Pedagogía Crítica. (pp. 45-66). Madrid, España: Editorial Popular.

Rivera, E. (1997). Identidad Nacional: La globalización e identidades en América Latina. Recuperado el 7 de abril de 2008, de /www.judithcorsino/valores-y-virtudes-parauna-peda.htm

Téllez, R.-López, G. (2002). Antropología, identidades y globalización. Elementos: Ciencia y cultura. Revista de Ciencia y Cultura, № 045, Vol. 9, pp. 19-23.

Zelaya, C. (1991). Educación cívica: los valores morales, cívicos y religiosos. San José, Costa Rica: EUNED. 